\title{
An optimisation scheme based on the local interaction simulation approach and Lamb waves for elastic property estimation in multi-layered composites
}

\author{
A.B. Spencer, K. Worden*, W.J. Staszewski, J.A. Rongong and N.D. Sims \\ Dynamics Research Group, Department of Mechanical Engineering, University of Sheffield, Sheffield, UK
}

\begin{abstract}
The paper demonstrates the application of guided ultrasonic waves for estimating material elastic properties in multi-layered composite plates. Two-dimensional Lamb wave propagation is modelled using the Local Interaction Simulation Approach (LISA). The wave responses are then used to estimate relevant material properties. This numerical inverse method is based on an optimisation scheme using a Differential Evolution (DE) algorithm. The method is demonstrated on a simulated seven-layered glass fibre-epoxy composite plate. The results show a good agreement between the exact and reconstructed material properties.
\end{abstract}

Keywords: Glass fibres, layered structures, mechanical properties, computational modelling, ultrasonics

\section{Introduction}

The inverse problem in wave propagation is one of the earliest inverse problems in Mechanical Engineering [1]. Various wave propagation elements can be reconstructed including boundaries, material properties, source and loading [2,3]. Application examples related to Non-Destructive Testing (NDT), ocean acoustics, earth and space exploration, biomedical examinations, radar control and solar astrophysics are given in [4].

It is well-known that wave propagation can be used to estimate the elastic properties of solids. In plate structures, the use of bulk ultrasound waves to obtain material properties is limited by the need to use wavelengths significantly shorter than the plate thickness. In contrast, Lamb waves are guided ultrasonic waves that propagate in the plane of the plate and therefore avoid this restriction. There is a vast literature related to the application of Lamb waves for the characterisation of different materials, as reviewed in [5,6]. The majority of these investigations study the dispersion characteristics of materials; once material properties are known, numerical modelling is used to obtain dispersion curves. The results are then validated using experimental phase measurements. The inverse problem, i.e. estimation of material properties from wave propagation, has attracted much less attention in the literature. Previous work in this area includes the application of direct phase velocity measurements in isotropic plates [7,8], concrete [9] and orthotropic composite plates [10,11]. The last-mentioned employed a non-linear least-squares scheme. Lamb wave responses in transversely isotropic composites [12] and dispersion curves in tri-layer structures [13] have also been used to estimate material properties. The latter used wave propagation modelling and an optimisation process to reconstruct the material properties. A method based on fitting the theoretical dispersion curves to ex-

\footnotetext{
*Corresponding author: K. Worden, Dynamics Research Group, Department of Mechanical Engineering, University of Sheffield, Mappin Street, Sheffield S1 3JD, United Kingdom. E-mail: k.worden@sheffield.ac.uk.
} 
perimental data in aluminium plates is given in the recent work [14]. The idea of inversion based on dispersion curves was exploited in a considerable body of work in the 1990s using measurements of leaky Lamb waves; the paper [15] marks an interesting point at which the technology was starting to make the transition to established procedure. A blind inversion scheme utilising fundamental Lamb wave mode velocities and a genetic algorithm has also been used to estimate material properties in a quasi-isotropic graphite-epoxy composite plate [16]. Although inversion based on dispersion curves has undeniably been successful, there are reported problems in dealing with some anisotropic media [17]. As well as dispersion curves, other experimental data which have been used for inversion with success are reflection/transmission spectra [11] and, more recently, slowness curves [18]. The latter reference also provides an example of the use of an evolutionary optimisation scheme in the form of a genetic algorithm. It appears that very little work has been performed utilising numerical modelling related to multilayered structures, dispersive media and guided waves. Recent work in this area includes application of new spectral layer elements [19]. Also, inversion based on simple waveform reconstruction appears to be very uncommon. The objective of the current paper is to use a full two-dimensional simulated Lamb wave displacement field and an optimisation scheme in order to establish material properties of multi-layered composites. The major novelty here is related to the application of the combined Local Interaction Simulation Approach (LISA) and Differential Evolution (DE) in order to carry out waveform reconstruction together with a Dynamic Time-Warping scheme which alleviates the problem of local minima encountered in all (nonlinear) optimisation problems. The structure of the paper is as follows. Section 2 briefly introduces the theory and implementation of LISA for the sake of completeness. The algorithm for material property estimation based on LISA and DE is presented in Section 3. Numerical analysis and results for a model glass fibre-epoxy seven-layer composite plate are given in Section 4. Finally, the paper is concluded in Section 5.

\section{Lamb wave modelling using the local interaction simulation approach}

This section gives a brief introduction to the propagation of Lamb waves in multiple layers and the two-dimensional Local Interaction Simulation Approach used to model these waves in multi-layered plates. In the classical sense, Lamb waves are guided waves propagating along a uniform thin plate with free boundaries [20]. The analytical analysis of Lamb wave propagation is rather a difficult problem for various reasons, dominant among these are the facts that the waves can propagate in potentially many modes and that the waves are dispersive, i.e. their velocities of propagation depend on their frequencies. This problem becomes even more complex when the waves propagate in multiple layers; in fact it is not immediately clear to the authors that such waves should truly be called Lamb waves; however, this terminology will be adopted throughout this paper for simplicity.

For the $N$-layered plate, shown schematically in Fig. 1, the Lamb wave equations can be derived from Navier's displacement equations of motion,

$$
\mu^{n} \nabla^{2} w^{n}+\left(\lambda^{n}+\mu^{n}\right) \nabla\left(\nabla \cdot w^{n}\right)=\rho^{n} \frac{\partial^{2} w^{n}}{\partial t^{2}}
$$

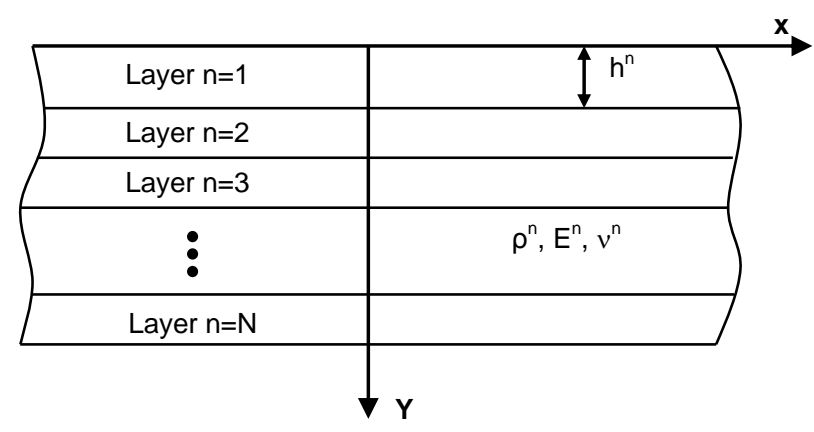

Fig. 1. Schematic illustrating the geometry of the $N$-layered composite plate. 
where $\rho$ is the density, $w^{n}=\left(w_{1}^{n}, w_{2}^{n}, w_{3}^{n}\right)$ is the three-dimensional displacement vector in the $n$th layer, $\lambda^{n}$ and $\mu^{n}$ are the first and second Lamé constants for layer $n$. The coordinate directions 1 and 2 correspond to $x$ (the in-plane direction) and $y$ (the through-thickness direction) in Fig. 1; the associated displacements are $w_{1}^{n}$ and $w_{2}^{n}$. The method of potentials transforms the displacements in Eq. (1) into field variables using the Helmholtz decomposition. This results in uncoupled equations describing the propagation of longitudinal and shear waves characterised by velocities $c_{L}^{n}$ and $c_{T}^{n}$ respectively When the physical displacements are recovered from the field variables, traction components, calculated from Hooke's law, generate the boundary conditions for the top and bottom free layers and it is the enforcing of these boundary conditions that gives Lamb waves. In addition, continuity conditions are required for the interfaces between each of the $N$ layers. All the boundary and continuity conditions can be written together in matrix form as,

$$
\left[A\left(\omega, k, \lambda^{n}, \mu^{n}, h^{n}\right)\right] \cdot\left[C^{n}\right]=[0]
$$

where $\omega=2 \pi f$ is the circular frequency of the wave, $k$ is the wave number, $h^{n}$ is the thickness of layer $n$ and the $A$ 's are individual matrix elements identified as factors of the respective $C^{n}$ unknowns from the set of boundary and continuity equations. Equation (2) can then be used to obtain the dispersion equations for the analysed plate by setting the determinant of $[A]$ to zero; the resulting condition relates the frequency $\omega$ to wavenumber $k$ for a given material (i.e. $\lambda^{n}, \mu^{n}$ ) and geometry (i.e. $h^{n}$ ) of the plate. The classical Rayleigh-Lamb wave propagation problem refers to determining these dispersion curves for given material properties and geometry of the plate. In the inverse problem, material properties can be obtained from the known geometry and estimated (i.e. simulated or experimental) dispersion curves, as demonstrated in [7-17]. The current paper uses direct wave propagation modelling for this task, which allows potential simplifications in the requirements of experimental instrumentation.

Any number of different numerical computational techniques can be used for the analysis of wave propagation in general; guided waves are usually more difficult to model than bulk waves. Both finite difference equations and finite element analysis have long histories and have been used widely for wave propagation in media with homogeneous or continuously varying physical properties. However, boundaries and discontinuities between different types of media lead to approximate solutions and can produce severe errors. The Local Interaction Simulation Approach (LISA) was proposed in the 1990s [21-23] to avoid these difficulties. The method is formally similar to the finite difference approach but it uses the so-called Sharp Interface Model (SIM) to impose the continuity, or in other words perfect contacts, of displacement and stresses at interfaces and discontinuities; this avoids the use of $a d$ hoc smoothing schemes. LISA is computationally efficient and has proven accurate for the modelling of wave propagation and diffusion problems in complex media [24,25], Lamb wave interaction with structural damage [26-28] and contact interfaces in nonlinear acoustics [29].

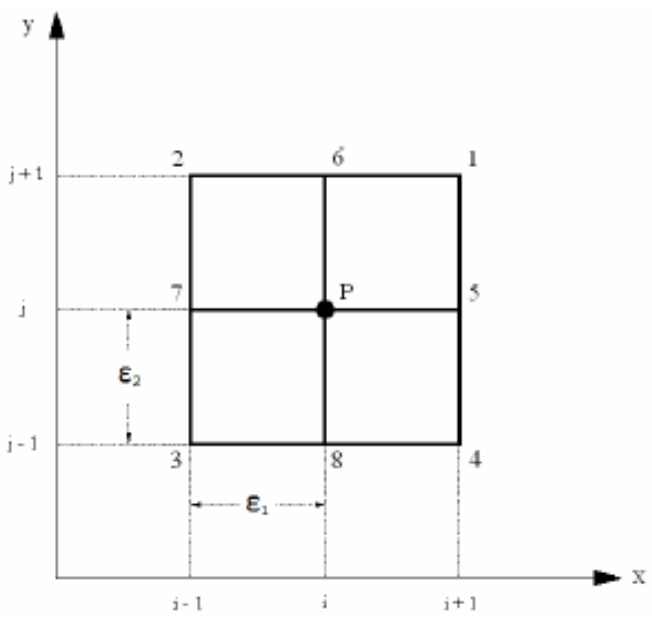

Fig. 2. Two-dimensional LISA discretisation model used for Lamb wave simulation. 
Equation (1) can be used as a starting point for the LISA-based wave propagation algorithm. In tensorial form, the equation is stated as (from now on, layer labels are suppressed),

$$
\partial_{l}\left(S_{k l m n} \partial_{n} w_{m}\right)=\rho \ddot{w}_{k} \quad(k, 1, m, n=1,2)
$$

where $S$ is the stiffness tensor, $\rho$ is the material density and the $w_{k}$ are the components of the particle displacement vector. Ignoring antiplane shear waves to concentrate on two-dimensional wave propagation, and assuming a homogenous specimen this can be simplified to,

$$
\sigma_{k} w_{k, k k}+\mu w_{k, h h}+v w_{h, k h}=\rho \ddot{w}_{k}, \quad(k=1,2, h=1,2)
$$

where $\sigma_{k}=S_{k k k k}, \lambda=S_{1122}, \mu=S_{1212}$ and $v=\lambda+\mu=\sigma-\mu$; a comma preceding a subscript denotes differentiation with respect to that variable. $\lambda$ and $\mu$ are, as before, the Lamé constants for the material. The various equations can be assembled into the matrix form,

$$
A W_{, 11}+B W_{, 22}+C W_{, 12}=\rho \ddot{W}
$$

where:

$$
A=\left(\begin{array}{cc}
\sigma_{1} & 0 \\
0 & \mu
\end{array}\right), B=\left(\begin{array}{cc}
\mu & 0 \\
0 & \sigma_{2}
\end{array}\right), C=\left(\begin{array}{ll}
0 & v \\
v & 0
\end{array}\right) \text { and } W=\left(\begin{array}{l}
w_{1} \\
w_{2}
\end{array}\right) .
$$

In a two-dimensional LISA simulation, the structure is discretised into cells as shown in Fig. 2. Cell labels will be given in brackets with cell (1) top right in Fig. 2 and cells (2), (3) and (4) following in the anti-clockwise sense. Each nodal point $P$ is at the junction of four cells. The second time derivatives across the four cells are required to converge towards a common value at the point $P$; this ensures that if the cell displacements are continuous at $\mathrm{P}$ for the two initial sampling instants at times $t=0$ and $t=1$, they will remain continuous for all later times. If one now allows heterogeneous materials, the various quantities associated with a cell $(c)$ will be identified by a superscript e.g. $\mu^{(c)}$.

Using a finite difference scheme for the spatial first derivatives in the four surrounding cells to $P$ gives four equations in eight unknown quantities $\left\{w_{m, n}\right\}, m=1, \ldots, 4, n=1,2$. These equations are omitted here for space reasons. Imposing continuity of the stress tensor $\tau$ at the point $P$ and using further finite difference formulae gives four additional equations in the unknown quantities $\left\{w_{m, n}\right\}$, thus allowing these unknown spatial first derivatives to be solved.

The following definitions are made,

$$
g^{(c)}=\lambda^{(c)}-\mu^{(c)}
$$

$\sigma_{k}=S_{k k k k}$ is redefined as the quantity averaged over the four cells neighbouring point P.

$$
\sigma_{k}=\frac{\sigma_{k}^{(1)}+\sigma_{k}^{(2)}+\sigma_{k}^{(3)}+\sigma_{k}^{(4)}}{4}
$$

Similarly, one redefines the symbols $\mu$ and $\rho$ as the averaged quantities,

$$
\mu=\frac{\mu^{(1)}+\mu^{(2)}+\mu^{(3)}+\mu^{(4)}}{4}
$$

and,

$$
\rho=\frac{\rho^{(1)}+\rho^{(2)}+\rho^{(3)}+\rho^{(4)}}{4}
$$


Finally, for convenience, one defines,

$$
\sigma_{5}=\frac{\sigma_{1}^{(1)}+\sigma_{1}^{(4)}}{2}, \sigma_{6}=\frac{\sigma_{2}^{(1)}+\sigma_{2}^{(2)}}{2}, \sigma_{7}=\frac{\sigma_{1}^{(2)}+\sigma_{1}^{(3)}}{2}, \sigma_{8}=\frac{\sigma_{2}^{(3)}+\sigma_{2}^{(4)}}{2}
$$

The particle displacements in the $x$ and $y$ directions in Fig. 1 will now be redenoted as $u$ and $v$ respectively. Recalling that $v^{(c)}=\lambda^{(c)}+\mu^{(c)}$, after a certain amount of algebra, discrete-time update equations for the $u$ and $v$ displacements at the point $P$ can be calculated as,

$$
\begin{aligned}
u_{t+1}= & 2 u_{t}-u_{t-1} \\
& +\frac{1}{\rho}\left[\sigma_{5} u_{5}+\sigma_{7} u_{7}+\mu_{6} u_{6}+\mu_{8} u_{8}-2\left(\sigma_{1}+\mu\right) u_{t}+\frac{1}{4} \sum_{c=1}^{4}(-1)^{c} v^{(c)} v_{t}\right. \\
& \left.-\frac{1}{4} \sum_{c=1}^{4}(-1)^{c} v^{(c)} v_{c}+g_{5} v_{5}+g_{6} v_{6}+g_{7} v_{7}+g_{8} v_{8}\right] \\
v_{t+1}= & 2 v_{t}-v_{t-1} \\
& +\frac{1}{\rho}\left[\mu_{5} v_{5}+\mu_{7} v_{7}+\sigma_{6} v_{6}+\sigma_{8} v_{8}-2\left(\sigma_{2}+\mu\right) v_{t}+\frac{1}{4} \sum_{c=1}^{4}(-1)^{c} v^{(c)} u_{t}\right. \\
& \left.-\frac{1}{4} \sum_{c=1}^{4}(-1)^{c} v^{(c)} u_{c}-g_{5} u_{5}-g_{6} u_{6}-g_{7} u_{7}+g_{8} u_{8}\right]
\end{aligned}
$$

where $t$ is the index associated with the time instant and new quantities are introduced: $g_{5}=\frac{1}{2}\left(g^{(4)}-g^{(1)}\right)$, $g_{6}=\frac{1}{2}\left(g^{(1)}-g^{(2)}\right), g_{7}=\frac{1}{2}\left(g^{(2)}-g^{(3)}\right)$ and $g_{8}=\frac{1}{2}\left(g^{(3)}-g^{(4)}\right)$. The displacements defined $\left(u_{k}, v_{k}\right)$ with $k=$ $1, \ldots, 8$, are those associated with the nodes labelled 1, .., 8 in Fig. 2 at time $t$ (for notational convenience, the $t$ label is suppressed in the equations; this is keeping with the original reference [22]).

Equations (11) and (12) are the principal displacement equations of LISA in two dimensions. A more detailed derivation can be found in [22], although note that a number of small errors present in the formulae within that paper have been corrected in Eqs (11) and (12). These two equations rely solely on known material properties $\rho, \lambda$ and $\mu$ for each cell and arbitrary discretisation steps, both spatial and temporal. They can easily be encoded and swiftly processed by a computational scheme such as Java or MATLAB, allowing LISA to be an effective simulation algorithm for wave propagation. A LISA software package with a graphical user interface has been created at the University of Sheffield using Java and this software has been used for the work here.

\section{Material property estimation: Algorithm and implementation}

\subsection{Lamb wave inverse problem}

Sections 2.1 and 2.2 have outlined the theory and modelling behind the forward problem for Lamb wave propagation in multiple-layered plates. In principle, the inverse Lamb wave problem allows one to obtain material properties for given geometry and dispersion characteristics. The latter can be obtained using numerical simulations or experimentally. The current work utilises the numerical model of wave propagation described in Section 2. The inversion is possible because the longitudinal $c_{L}^{n}$ and shear $c_{T}^{n}$ wave velocities can be related directly to material constants of interest for each layer $n$ of the composite plate by $[27,30]$, 


$$
\begin{gathered}
v^{n}=\frac{1-2\left(\frac{c_{T}^{n}}{c_{L}^{n}}\right)^{2}}{2-2\left(\frac{c_{T}^{n}}{c_{L}^{n}}\right)^{2}} \\
E^{n}=2 \rho^{n}\left(1+v^{n}\right)\left(c_{T}^{n}\right)^{2}
\end{gathered}
$$

Rather than make direct use of the formulae above (or more complicated formulations based on dispersion curves etc.), the current paper takes a slightly more indirect approach. The LISA algorithm is used to model the forward propagation of the waves in a multi-layered plate for fixed values of the material properties. The predicted waveform can then be compared to an 'experimentally obtained' waveform and a prediction error can be computed. If the LISA module is then embedded within an optimisation scheme, the material properties can be adjusted in accordance with the scheme until the prediction error is minimised. As this paper is essentially concerned with 'proof of concept', the 'experimental' target waveform is also generated by the LISA algorithm in order that the true results for the material properties are known. In order to keep the complexity of the problem within reasonable bounds, the example plate is composed of only two different materials; however, these materials are alternated in a seven-layer structure to keep the sample of engineering interest. The sample here represents a glass fibre-epoxy laminate. As a further simplification in the spirit of this proof-of-concept investigation, a two-dimensional propagation model is chosen. This is not because three-dimensional models are impossible; it is because they are considerably more time-consuming and make the optimisation approach prohibitive on a standard PC computer. This restriction means that the (generally) anisotropic behaviour of composite laminates is not captured; each material has essentially one longitudinal and transverse wave velocity. This restriction will be lifted in later studies.

\subsection{The optimisation scheme based on differential evolution}

Following the algorithm described in Section 3.1, the Differential Evolution (DE) optimisation scheme was used to match the predicted responses with trial wave propagation parameters, with numerically simulated 'target' responses. (In practise, experimental responses would clearly be used.) DE is an optimisation technique which belongs to the class of evolutionary algorithms. The method is descended from (as are most evolutionary algorithms) the Genetic Algorithm (GA) and was first proposed in [30]. Both the GA and DE algorithms attempt to find a globally optimal solution to a given problem, avoiding local extrema as far as possible. What differentiates DE from GAs is the parameter domain, which in the case of DE is real-valued. Therefore the DE procedure is used to optimise real-valued problems.

Mathematically, if the optimisation problem is to find a minimum, then for a given objective function $f: X \subseteq \mathfrak{R}^{D}$ the DE attempts to find $x^{*} \in X$ such that $f\left(x^{*}\right) \leqslant f(x) \forall x$. In order to solve this problem, the DE transforms a randomly generated initial population of parameters into an optimal solution through an iterated cycle of genetic operations such as mutation, crossover and selection; the details can be found in [30].

For the problem investigated in the current paper, the objective function is constructed as follows. Because the plate is composed of alternate layers of two materials; the parameter vectors for optimisation consist of 6 real numbers: the density and longitudinal and transverse wave velocities for each of the two materials i.e. $\left\{\rho_{1}, c_{T 1}, c_{L 1}, \rho_{2}, c_{T 2}, c_{L 2}\right\}$. To obtain a cost value, these parameters are used in the LISA simulation to predict the displacement wave $\hat{w}_{t}$ response at a point on the plate corresponding to the point where the 'true' waveform $w_{t}$ has been recorded. A mean-squared error can then be computed from the sampled values of the waveforms,

$$
E=\sum_{t=1}^{N}\left(w_{t}-\hat{w}_{t}\right)^{2}
$$

and it is this 'cost' which is minimised in the optimisation strategy. For the DE algorithm, two parameters are needed: $F$ is essentially a mutation parameter and $C R$ is a crossover probability. Parameter values $F=0.9$ and $C R=$ 
0.5 were used; these values having proved useful in previous problems. The population was set to 60; again previous work had established that 10 times the number of parameters worked well in general. The property values in the initial population were randomly generated on fixed ranges with endpoints one order of magnitude above and below their true values. The procedure was terminated after 250 generations; this stopping criterion was chosen to terminate the procedure relatively early to allow many runs of the DE to take place. As the optimisation strategy is stochastic, best practice demands that, in order to improve the possibility of finding the global minimum for the problem under investigation, a large number of runs are made with different initial populations.

\subsection{Dynamic time warping}

The objective function, described in Section 3.2, could potentially require the comparison of Lamb wave responses which are not necessarily aligned in time, although this is not the case here. A more serious problem here is that the cost function is likely to have many local minima as a result of the target and candidate waveforms being shifted with respect to each other by a whole number of wave cycles. Therefore Dynamic Time Warping (DTW) [31] was used to minimise the effects of possible misalignment. The DTW approach measures similarities between two time responses which vary in time or speed. The time scale of one signal is shifted against the other by varying amounts along the length of the signal. This enables signals that are somewhat out of phase but otherwise similar to be given a low error value. In the same situation, a straightforward mean squared error would give a high error value.

The effect of DTW is illustrated in Fig. 3 which shows how a candidate signal (red) when compared with the target signal (blue), would have matching parts shifted in time to overlay regions of the target signal. This would lead to a low error value when the two signals are compared. However a small error value is added to penalise the amount of shifting used, so that an exact match (without any shifting required) would give an even lower error.

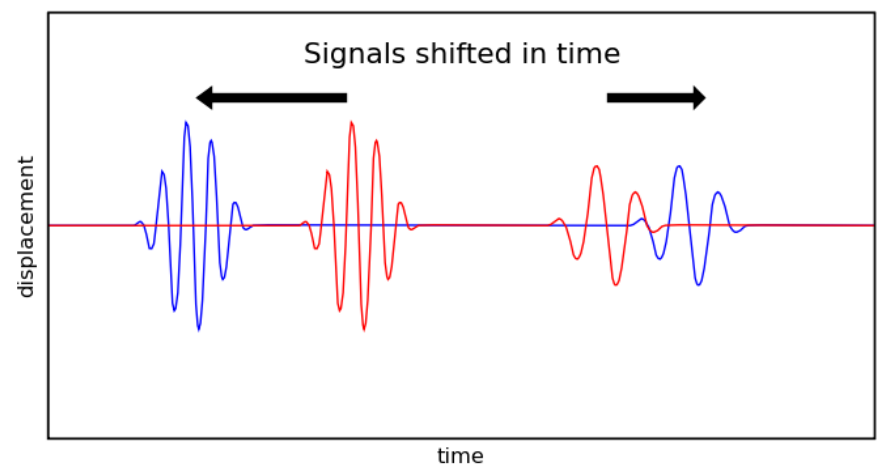

Fig. 3. Diagram showing how parts of a waveform are time shifted to overlay matching parts of the waveform being compared.

The DTW algorithm used builds up a matrix of error values by comparing the value - for each iteration - from the candidate signal with each value from the target signal (an interpolation step is needed here). This gives a matrix of error values; the line along the diagonal indicates the error for the unshifted comparison, and the various paths away from the diagonal indicate different combinations of insertions (expanding time) and deletions (compressing time). Figure 4 shows a portion of such a matrix represented as shades of grey with lighter shades corresponding to lower error (or cost). The diagonal line shows the route of an unshifted comparison, while the adjacent wandering line shows the lowest cost path found by the DTW algorithm.

Figure 5 shows an example of the DTW procedure applied to Lamb wave response signals. The light grey line shows the 'exact' wave propagation model signal that needs to be matched. The dark grey line shows a candidate simulated signal and the black line shows the candidate signal time-warped to give the best match possible. The 'clipping' in Fig. 5 is the result of a stretching distortion introduced by the DTW procedure; such distortions only occur in the early stages of the optimisation; as the algorithm approaches the global minimum it disappears. 


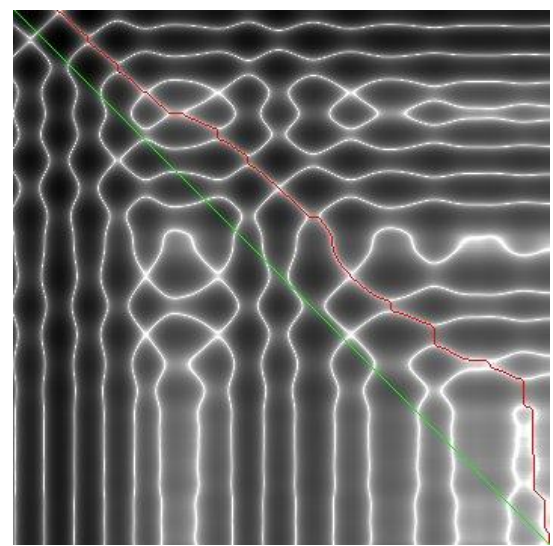

Fig. 4. Example of a portion of the time warping cost matrix.

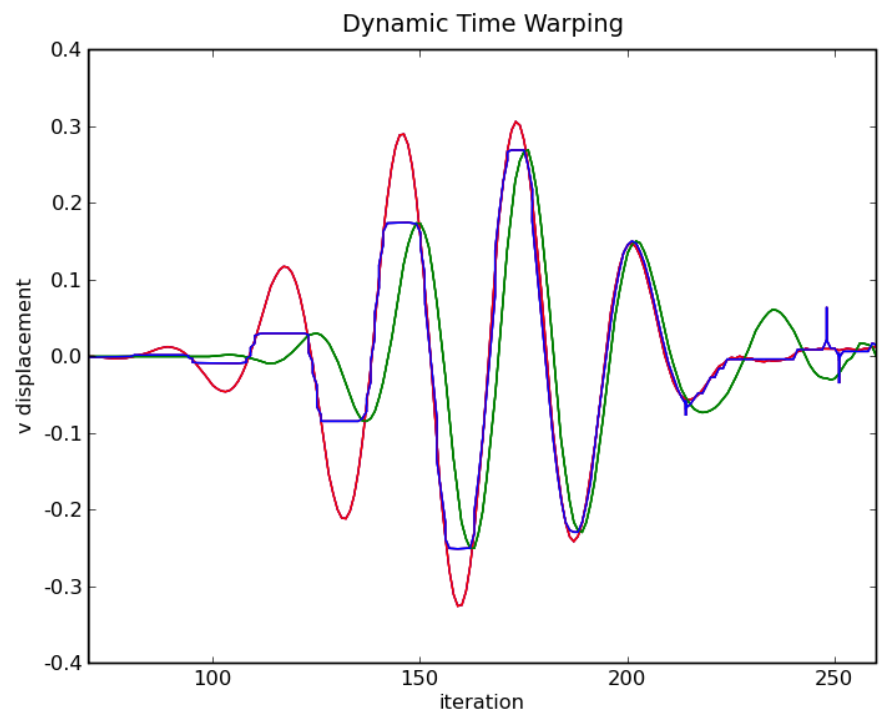

Fig. 5. Example showing the use of dynamic time warping for Lamb wave response signals.

\section{Numerical results and discussion}

As discussed above, the algorithm for material property reconstruction was tested using an example of a glass fibre-epoxy composite plate consisting of seven layers. The thickness of each layer was $1 \mathrm{~mm}$. Figure 6 shows the two-dimensional model of the composite plate, showing the arrangement of the layers of the two materials and the position of the actuator and sensor. The model was surrounded by a border of air, introduced to enable the correct calculation of reflections at the air/glass interface at the surfaces of the plate. Material properties chosen in the wave propagation model were as follows:

\begin{tabular}{llll}
\hline \multicolumn{1}{c}{ Property } & Units & Glass fibre & \multicolumn{1}{c}{ Epoxy } \\
\hline density & $\mathrm{g} / \mathrm{mm}^{3}$ & 0.00244 & 0.00121 \\
longitudinal velocity & $\mathrm{mm} / \mathrm{s}$ & 5546000 & 2724000 \\
transverse velocity & $\mathrm{mm} / \mathrm{s}$ & 3455000 & 1157000 \\
equivalent Young's modulus & $\mathrm{GPa}$ & 68.9 & 4.50 \\
equivalent Poisson's ratio & & 0.183 & 0.390 \\
\hline
\end{tabular}




\begin{tabular}{|l|}
\hline Glass fibre \\
\hline Epoxy \\
\hline Glass fibre \\
\hline Epoxy \\
\hline Glass fibre \\
\hline Epoxy \\
\hline Glass fibre \\
\hline
\end{tabular}

Fig. 6. The model of a composite plate used in the LISA simulation.
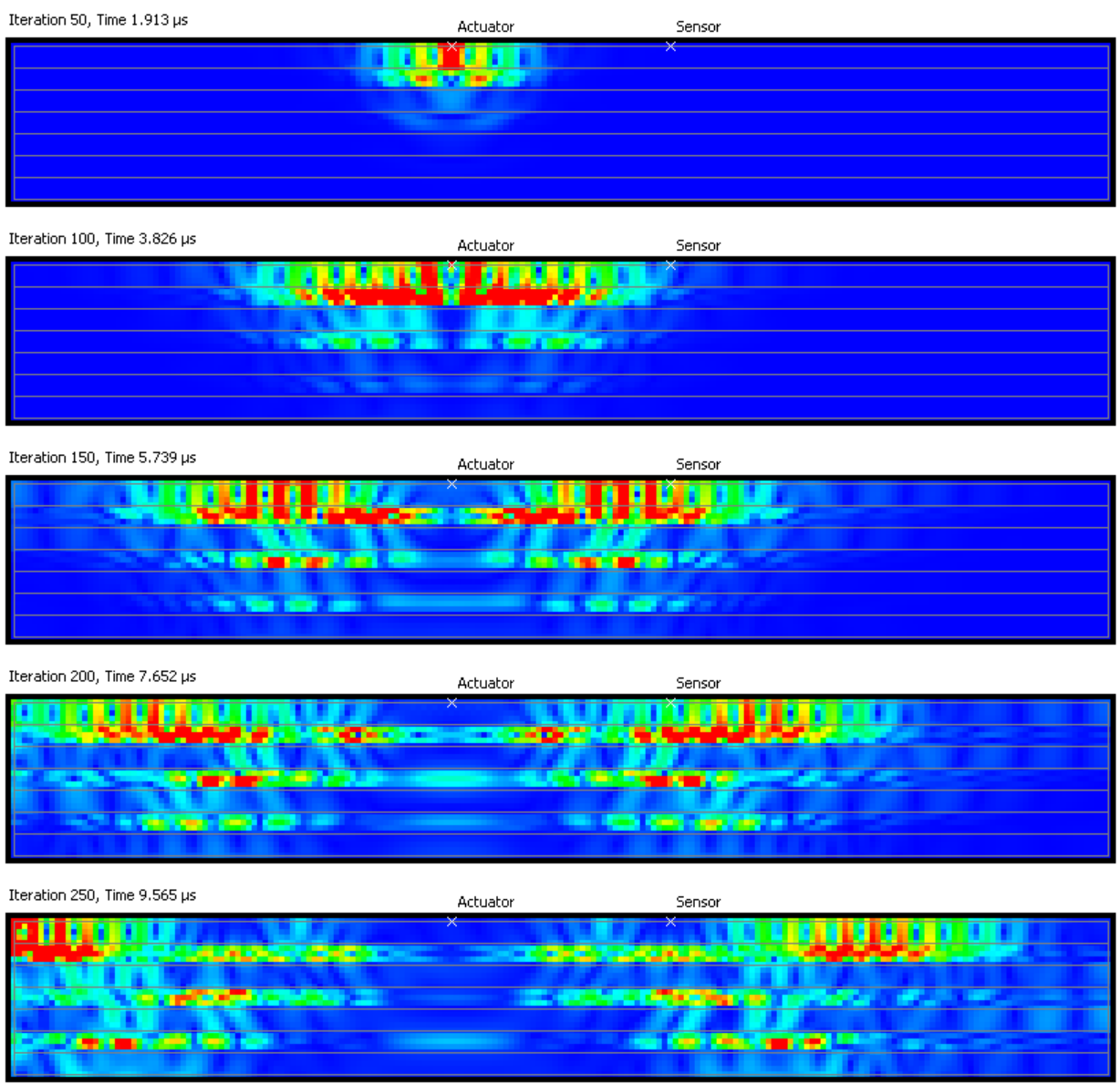

Fig. 7. Displacement vector magnitude in the 2D wave model of the composite plate after 50, 100, 150, 200, and 250 iterations (time steps).

The 2D model of the plate was $7 \mathrm{~mm}$ overall thickness and $50 \mathrm{~mm}$ long. The simulation covered a period of $10 \mu \mathrm{s}$ from the onset of the actuation signal. In reality, an experimental procedure would launch and receive the waves using actuators with physical properties of their own which should be modelled. To simplify matters here, point actuation and sensing at nodes of the model mesh are assumed. The $50 \mathrm{~mm}$ length was chosen to be sufficient to ensure that significant reflections from either end of the model did not reach the 'sensor' during the period of analysis, so that the simulation was equivalent to that for an infinite plate. The model was discretised into $0.25 \mathrm{~mm}$ square cells, giving a total of 5600 elements ( 28 by 200). LISA was used to simulate wave propagation between the actuator and sensor as shown in Fig. 7. The actuator and sensor were positioned $10 \mathrm{~mm}$ apart at the top of the plate. Five cycles of a sine wave with a Hanning window envelope were introduced into the plate as a time-varying dis- 
placement profile perpendicular to its surface at the actuation point. The frequency of the excitation was $1000 \mathrm{kHz}$ and the amplitude was taken as 1 unit, a reference value for subsequent values of the modelled displacements.

Figure 7 shows waves propagating through the two-dimensional model of the analysed composite plate at iterations 50,100, 150, 200 and 250. The waves can be observed from the colour indicating the magnitude of the displacement vector at each node. Figure 8 shows the simulated Lamb wave response generated by propagation through this model using the target parameters. This response was used as the target vector for the DE optimisation scheme in the Lamb wave inverse problem.

The DE optimisation was performed a total of 82 times. Of these, 20 runs identified the four velocity parameters $\left\{c_{T 1}, c_{L 1}, c_{T 2}, c_{L 2}\right\}$ to a fairly good approximation and 3 of these runs gave excellent agreement. The optimisation was unable to match the absolute values of the densities, but where the velocity parameters matched, the ratio of the two densities also gave good agreement. This would be sufficient if the overall size and weight of the plate are known. If the DE had been allowed to run for more generations, it is expected that more of the 20 good runs would have converged very closely to the expected values, but a 250 generation limit was imposed (as discussed earlier) because of time constraints. The other $62 \mathrm{DE}$ optimisation runs which showed bad agreement with the expected values, appear to have become trapped in local minima, and it is unlikely that running the DE for more generations would have helped in this case. The fact that some optimisation runs end at local minima is not considered an issue here as the algorithm is not so computationally expensive that many runs are precluded.

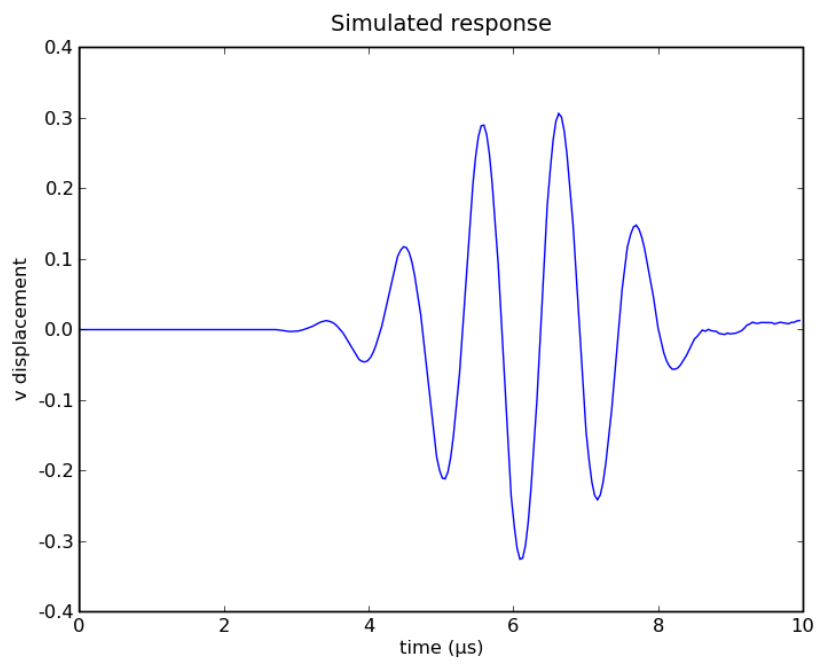

Fig. 8. Example of Lamb wave response from the analysed LISA model of the composite plate.

The lowest error value was $3.41 \times 10^{-6}$. Because the error function (15) is not normalised, the lowest value obtained has no immediate meaning, it should be understood in the context of the accuracy of the parameter estimates. The optimised parameters from the best run were (in the units used at the beginning of the section):

\begin{tabular}{cll}
\hline Value & Identified & Expected \\
\hline$\rho_{1}$ & 0.07092 & 0.00244 \\
$\mathrm{vL}_{1}$ & 5545246 & 5546000 \\
$\mathrm{vT}_{1}$ & 3453564 & 3455000 \\
$\rho_{2}$ & 0.03515 & 0.00121 \\
$\mathrm{vL}_{2}$ & 2723157 & 2724000 \\
$\mathrm{vT}_{2}$ & 1158006 & 1157000 \\
\hline
\end{tabular}

As observed above, the absolute densities do not agree, but the ratio of the densities $\rho_{1 /} \rho_{2}=2.0179$ is very close to the expected value of 2.0165. This approach is unable to find the absolute values for the densities, because the 
output waveform is not sensitive to the individual values, only the relative densities. The reflections from the surface of the plate would be slightly affected by the relative density of glass fibre and air, but because air is so much lighter, the effect makes very little difference to the output waveform. Also, in the simulation that was used, the actuation impulse was applied as an absolute displacement at the actuation point. If the actuation signal was treated as a force applied at that point, then the density of the glass fibre layer would have a large effect on the overall amplitude of the output signal, and in this case it is expected that the values of the densities would be determined by the procedure. The reason that an applied displacement was prescribed here was that a transducer model would have been desirable in prescribing a force. Transducer models were not included in the two-dimensional implementation of LISA used here, but are being included in the full three-dimensional implementation currently under development.

The results shown presented above are for numerically simulated data uncorrupted by any noise. In order to investigate the robustness of the algorithm, it is useful to see how it performs in the presence of noise. To do this, Gaussian noise of variance $10 \%$ of the signal variance for the pulse in Fig. 8 was added and the optimisation was performed once more. The noisy signal for reconstruction is shown in Fig. 9.

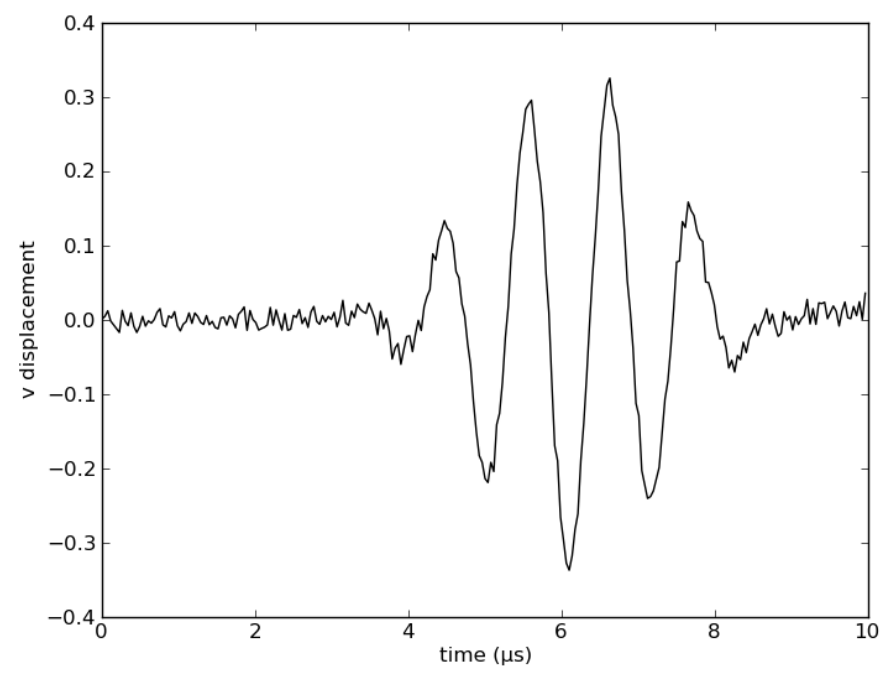

Fig. 9. Effect of adding 10\% Gaussian noise for target signal for reconstruction by LISA.

Note that this represent severe noise in the context; typical Lamb wave profiles acquired experimentally would be averaged over many samples and the instrumentation and sensors usually ensure that signal-to-noise ratio is high for a single sample anyway. For the case of the noisy data, 10 optimisation runs were carried out with different initial populations; of these only one of the optimisations found the correct minimum. However, the results were very good, the parameter estimates being given below.

\begin{tabular}{cll}
\hline Value & Identified & Expected \\
\hline$\rho_{1}$ & 0.100489 & 0.00244 \\
$\mathrm{vL}_{1}$ & 5309645 & 5546000 \\
$\mathrm{vT}_{1}$ & 3596693 & 3455000 \\
$\rho_{2}$ & 0.051092 & 0.00121 \\
$\mathrm{vL}_{2}$ & 2741265 & 2724000 \\
$\mathrm{vT}_{2}$ & 1150606 & 1157000 \\
\hline
\end{tabular}

The ratio of densities is 1.967 , compared to the true value of 2.017 . The quality of the reconstructed signal with the properties above is clearly shown in Fig. 10. This exercise gives some confidence that the approach is not sensitive to signal noise. 


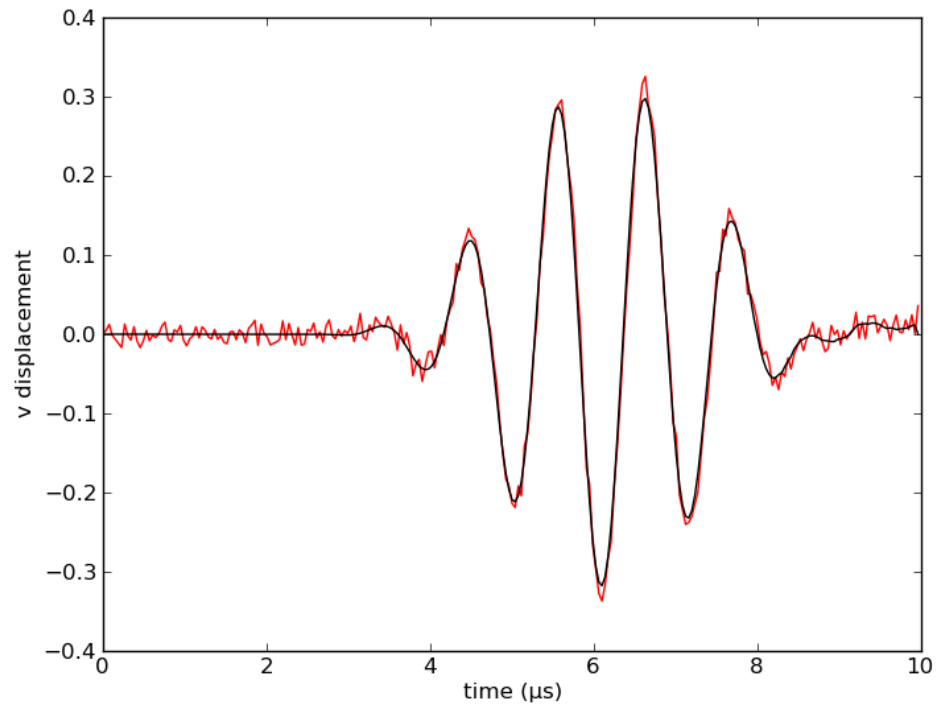

Fig. 10. Target signal (red line) compared with reconstruction by LISA (black).

\section{Discussion and conclusions}

The LISA modelling of Lamb wave propagation has been used as part of an optimisation strategy to reconstruct material properties in a multi-layered glass fibre-epoxy composite plate. A simple approach based on the time-history of the waveform is used which offers potential simplifications in the required instrumentation for inversion. The results show, on a much simplified synthetic data set, that this approach has clear potential for identifying material properties within multi-layered composite structures.

A first observation relates to the actuation and sensing of the waves in the simulation model. An initial attempt at the inversion used a pulse-echo strategy through the thickness of the plate. The obvious advantage of this was that the LISA model reduced to one-dimensional wave propagation. It was clear from this initial study that actuation and sensing needed to be done at separate locations to allow sufficient information to be gathered about the internal structure. Because the problem then became propagation in a plate, it was assumed that the mode of propagation would be by Lamb waves. This would be expected given the relationship between the wavelength and the thickness of the plate here (wavelength of the order of the overall thickness and proportionally greater than the thickness of individual layers). As the actuator-sensor distance here is only approximately 1.3 times the overall plate thickness it may be unlikely that full Lamb wave propagation is established; however, Fig. 7 shows very clearly that the propagation is by guided waves and the results of the inversion show that the increased complexity of the propagation allows the waveform to carry enough information to fix the material properties. The exception to latter statement is the overall density of the plate, although the density ratio between the two materials was estimated accurately; density has always presented problems [15], and the matter will clearly bear further thought. In terms of the optimisation strategy, the dynamic time warping approach applied here appears to be necessary in order for the optimisation scheme to effectively avoid local minima.

A very preliminary investigation shows that the approach does not appear to be very sensitive to signal noise.

An important extension of the current work will need to take the simulations and optimisation to three dimensions in order to allow the identification of the material properties of anisotropic composite laminates.

In general when pursuing an inverse problem approach of the type presented here, one would wish to carry out a more general sensitivity analysis in order to determine which material properties are most responsible for accurate signal reconstruction. One would also wish to investigate the use of weighted cost functions or regularisation schemes. However, this is not presented here as the paper was intended mainly as a proof of concept and the ap- 
proach worked quite well using an unweighted least-squares cost function in that context. The more rigorous analysis will be carried out in the context of the three-dimensional analyses planned for the future.

Finally, it should be mentioned that material property reconstruction by an optimisation or inverse approach is not in any way restricted to the use of wave propagation data. There is a developed literature associated with material property estimation for composite structures based on modal data, the reference [33] provides an early example of such an approach. However, it is not within the remit of the current paper to make a principled comparison between the modal and wave-based methods.

\section{Acknowledgments}

The authors would like to express their gratitude to Dr James Hensman of the University of Sheffield for useful discussions and for suggesting the use of the dynamic time warping procedure in the current investigations. The authors would also like to acknowledge a number of useful discussions on the nature of modelling wave propagation with Professor Paul Wilcox of the Department of Mechanical Engineering in The University of Bristol. Finally the authors would like to thank the anonymous referees for their constructive comments which led to improvements in the paper.

\section{References}

[1] J.D. Achenbach, Wave Propagation in Elastic Solids, Elsevier, 1973.

[2] F. Santosa, Y.H. Pap, W.W. Symas and C. Holland, Inverse Problems of Acoustic and Elastic Waves, SIAM, 1984.

[3] D. Colton and R. Kress, Inverse Acoustic and Electromagnetic Scattering Theory, Springer, Berlin, 1993.

[4] G.R. Liu and X. Han, Computational Inverse Techniques in Nondestructive Evaluation, CRC Press, 2003.

[5] D.E. Chimenti, Guided waves in plates and their use in materials characterization, Applied Mechanics Reviews 50 (2003), $247-284$.

[6] W.J. Staszewski, Structural Health Monitoring Using Guided Ultrasonic Waves, in: Advances in Smart Technologies in Structural Engineering, J. Holnicki-Szulc and C.A. Mota Soares, eds, Springer, 2004, pp.117-162.

[7] C.K. Jen, J.F. Bussiere, G.W. Farnell, E.L. Adler and M. Esonu, Elastic constant evaluation using dispersive property of acoustic waves, Reviews of Progress in Quantitative NDE 4 (1984), 889-900.

[8] W.P. Rogers, Elastic property measurement using rayleigh-lamb waves, Research in Nondestructive Evaluation 6 (1995), $185-208$.

[9] M. Sun, W.J. Staszewski, R.N. Swamy and Z. Li, Application of low-profile piezoceramic transducers for health monitoring of concrete structures, NDT\&E International 41 (2008), 589-595.

[10] S.I. Rokhlin, C.Y. Wu and L. Wang, Application of Coupled Ultrasonic Plate Modes for Elastic Constant Reconstruction of Anisotropic Composites, Reviews of Progress in Quantitative NDE 9B (1990), 1403-1410.

[11] S.I. Rokhlin and D.E. Chimenti, Reconstruction of elastic constants from ultrasonic reflectivity data in a fluid coupled composite plate, Reviews of Progress in Quantitative NDE 9B (1990), 1411-1418.

[12] M.R. Karim, A.K. Mal and Y. Bar-Cohen, Determination of the elastic constants of composites through inversion of leaky lamb wave data, Reviews of Progress in Quantitative NDE 9A (1990), 109-116.

[13] B. Bougaze, M. Sidiki and A. Ramadani, Guided ultrasonic propagation in the tri-layer structures. application to the reconstruction of their material properties from dispersion curves, European Physics Journal: Applied Physics 37 (2007), 223-228.

[14] J.L. Deán, C. Trillo, A.F. Doval and J.L. Fernández, Determination of thickness and elastic constants of aluminium plates from full-field wavelength measurements of single-mode narrowband lamb waves, Journal of the Acoustical Society of America 124 (2008), 1477-1489.

[15] Y. Bar-Cohen, M. Mal, S.-S. Lih and Z. Chang, Composite Materials Stiffness Determination and Defects Characterization Using Enhanced Leaky Lamb Wave Dispersion Data Acquisition Method, SPIE 6th Annual International Symposium on NDE of Aging Aircraft, Airports and Aerospace Hardware, Newport Beach, CA., 1999, pp. 3586-35.

[16] J. Vishnuvardhan, C.V. Krishnamurthy and K. Balasubramanian, Blind inversion method using lamb waves for the complete elastic property characterization of anisotropic plates, Journal of the Acoustical Society of America 125 (2009), 761-771.

[17] N.S. Rao, Inverse Problems in Ultrasonic Non-Destructive Characterization of Composite Materials Using Genetic Algorithm, MS Thesis, Mississippi State University, 1997.

[18] J. Vishnuvardhan, C.V. Krishnamurthy and K. Balasubramanian, Genetic algorithm based reconstruction of the elastic moduli of orthotropic plates using an ultrasonic guided wave single-transmitter-multiple-receiver SHM array, Smart Materials and Structures 16 (2007), $1639-1650$

[19] A. Chakraborty and S. Gopalakrishnan, Wave propagation in inhomogeneous layered media: Solution of forward and inverse problems, Acta Mechanica 169 (2004), 153-185.

[20] J.L. Rose, Ultrasonic Waves in Solid Media, Cambridge University Press, 1999.

[21] P.P. Delsanto, T. Whitcombe, H.H. Chaskelis and R.B. Mignogna, Connection machine simulation of ultrasonic wave propagation in materials I: One-dimensional case, Wave Motion 16 (1992), 65-80. 
[22] P.P. Delsanto, R.S. Schechter, H.H. Chaskelis, R.B. Mignogna and R. Kline, Connection machine simulation of ultrasonic wave propagation in materials II: Two-dimensional case, Wave Motion 20 (1993), 295-314.

[23] P.P. Delsanto, R.S. Schechter and R.B. Mignogna, Connection machine simulation of ultrasonic wave propagation in materials III: Three -dimensional case, Wave Motion 26 (1994), 329-339.

[24] V. Agostini, P.P. Delsanto, I. Genesio and D. Olivero, Simulation of lamb wave propagation for the characterization of complex structures, IEEE Transactions on Ultrasonics, Ferroelectrics, and Frequency Control 50 (2003), 441-448.

[25] B.C. Lee, M. Palacz, M. Krawczuk, W. Ostachowicz and W.J. Staszewski, Wave propagation in sensor/actuator diffusion bond model, Journal of Sound and Vibration 276 (2004), 671-687.

[26] B.C. Lee and W.J. Staszewski, Modelling of lamb waves for damage detection in metallic structures; Part II: Wave interaction with damage, Smart Materials and Structures 12 (2003), 804-814.

[27] B.C. Lee and W.J. Staszewski, Lamb wave propagation modelling for damage detection in metallic structures; Part I: Two-dimensional analysis, Smart Materials and Structures 16 (2007), 249-259.

[28] B.C. Lee and W.J. Staszewski, Lamb wave propagation modelling for damage detection in metallic structures; Part II: Damage monitoring strategy, Smart Materials and Structures 16 (2007), 260-274.

[29] S. Hirsekorn, M. Hirsekorn and P.P. Delsanto, Nonlinear Ultrasonic Transmission Through Thin Bonded Interfaces: Theoretical Background and Numerical Simulations, in: Universality of Nonclassical Nonlinearity, P.P. Delsanto, ed., Springer, 2007.

[30] R. Storn and R. Price, Differential evolution - a simple and efficient heuristic for global optimisation over continuous spaces, Journal of Global Optimization 11 (1997), 341-359.

[31] C.S. Myers and L.R. Rabiner, A comparative study of several dynamic time-warping algorithms for connected word recognition, The Bell System Technical Journal 60 (1981), 1389-1409.

[32] A.B. Spencer, K. Worden, S.G. Pierce, J.J. Hensman, D. Chetwynd and W.J. Staszewski, Force Characterisation of a Laser Impulse using Differential Evolution with a Local Interaction Simulation Algorithm, Proceedings of 23rd International Conference on Noise \& Vibration Engineering, Leuven, On CD, 2008.

[33] C.M. Mota Soares, M.M. Defreitas, A.L. Araujo and P. Pedersen, Identification of material properties of composite plate specimens, Composite Structures 25 (1993), 277-285. 

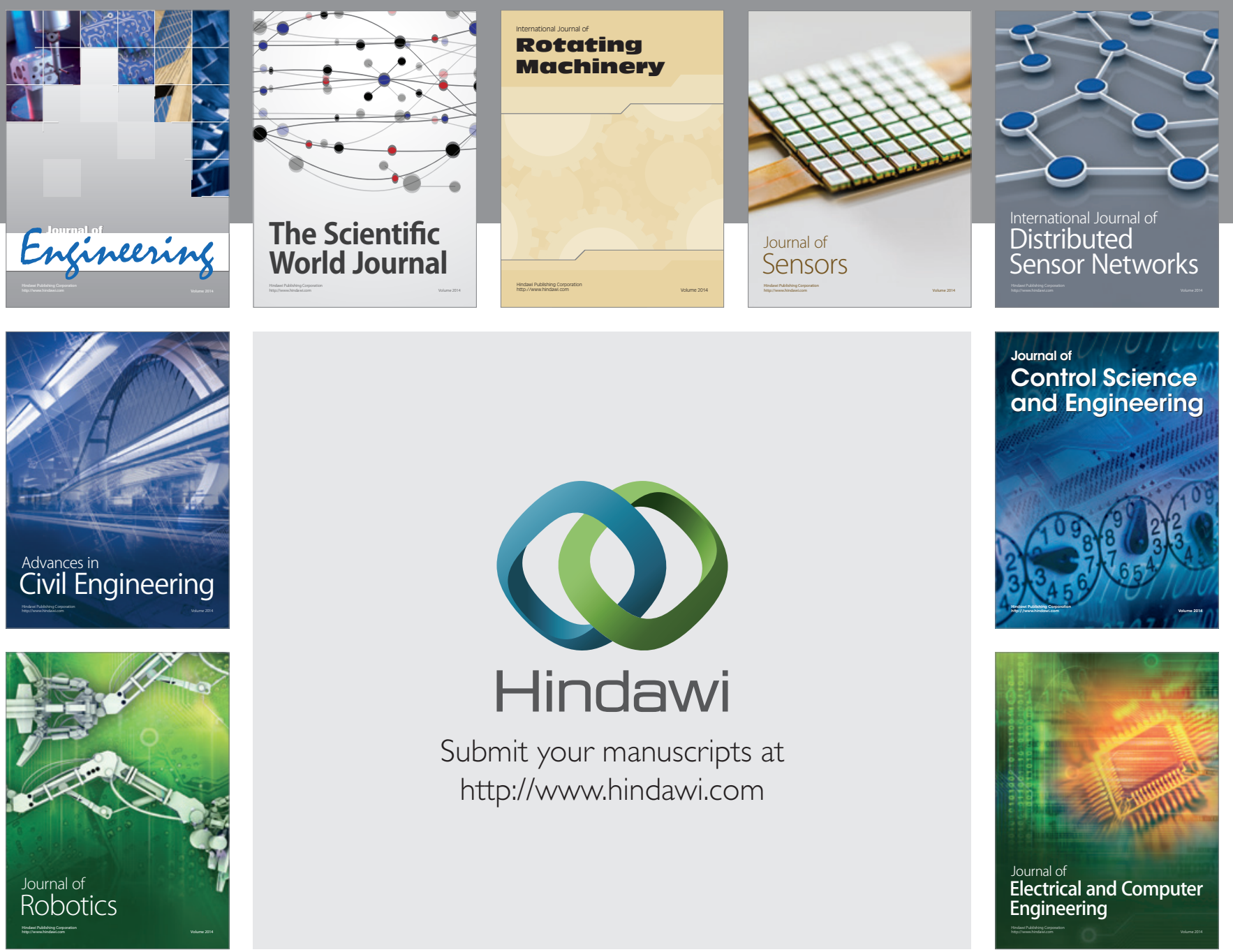

Submit your manuscripts at

http://www.hindawi.com
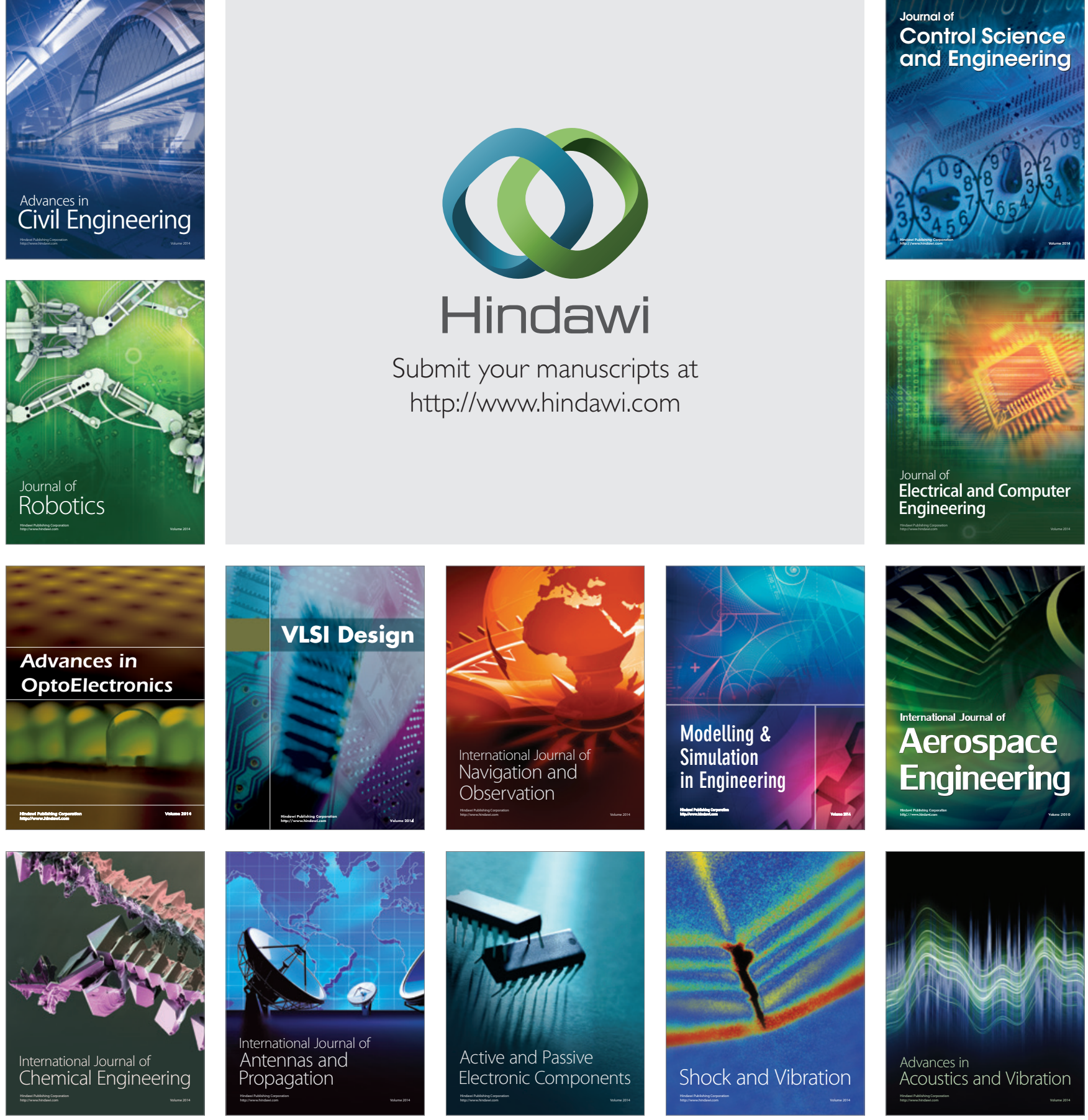